\title{
Design and Characterization of Polymeric Blends for Biomedical Applications
}

\author{
A. A. BARBA ${ }^{1}$, G. LAMBERTI ${ }^{2}$, L. RABBIA ${ }^{2}$, \\ M. GRASSI $^{3}$, G. GRASSI ${ }^{4}$, D. LAROBINA ${ }^{5}$ \\ ${ }^{1}$ Dipartimento di Scienze Farmaceutiche, Università di Salerno, Fisciano (SA), Italia \\ ${ }^{2}$ Dipartimento di Ingegneria Chimica e Alimentare, Università di Salerno, Fisciano (SA), Italia \\ ${ }^{3}$ Dipartimento dei Materiali e delle Risorse Naturali, Università di Trieste, Trieste, Italia \\ ${ }_{5}^{4}$ Dipartimento di Scienze della Vita, Università di Trieste, Trieste, Italia \\ ${ }^{5}$ Istituto per i Materiali Compositi e Biomedici, CNR, Portici (NA), Italia \\ E-mails: aabarba@unisa.it (A.A. Barba),glamberti@unisa.it (G. Lamberti), mario.grassi@dicamp.units.it \\ (M. Grassi), ggrassi@units.it (G. Grassi), larobina@unina.it (D. Larobina) \\ Sci Pharm. 2010; 78: 617 \\ doi:10.3797/scipharm.cespt.8.PDD28 \\ In biomedical applications, it should be useful to have a system easy to \\ administrate (the best would be a liquid injectable form), with structural \\ properties in physiological conditions (the best would be a soft gel), able to \\ resist to external stimulus (such us the erosion from body fluids, in this case the \\ surface should be a hard gel). Such a system could be used as a drug delivery \\ system, as well as a temporary scaffold. \\ From previous investigation, we found out that the acqueous solution of \\ Pluronic F127 and alginate (18\% F127 + 2\% alginate), is liquid at low \\ temperature (around $5^{\circ} \mathrm{C}$ ), it gives a soft gel due to the Pluronic thermogelation \\ at body temperature (around $37^{\circ} \mathrm{C}$ ), and it gives a hard gel after the exposition \\ at solutions of bivalent cations (reticulation, using $\mathrm{Ca}^{2+}, \mathrm{Cu}^{2+}$ ). \\ Prior of any other application, the system has to be thouroughly characterized. \\ Therefore, 1) the mechanical properties of the solution were investigated by

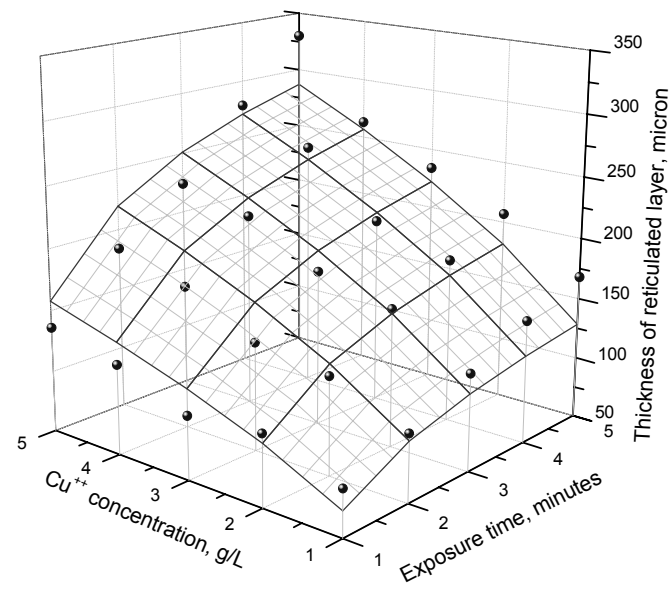 \\ using rotational rheometry (monitoring \\ the increase of solution moduli during \\ heating); 2) the compressive behavior \\ was investigated with impermeable and \\ porous parallel plates (underlining the \\ rule of the water to build up the \\ properties of the gel); 3) the kinetics of \\ cuprum diffusion and of hard gel \\ formation were quantified (obtaining the \\ thickness of reticulated layer - see the \\ Figure - as function of cuprum solution \\ concentrations and of exposure time. In \\ the Figure, the symbols are experimental \\ data and the surface curve is the model \\ prediction). Further investigations are still ongoing. The data gatered will be \\ used for the proper design of future biomedical applications.
}

